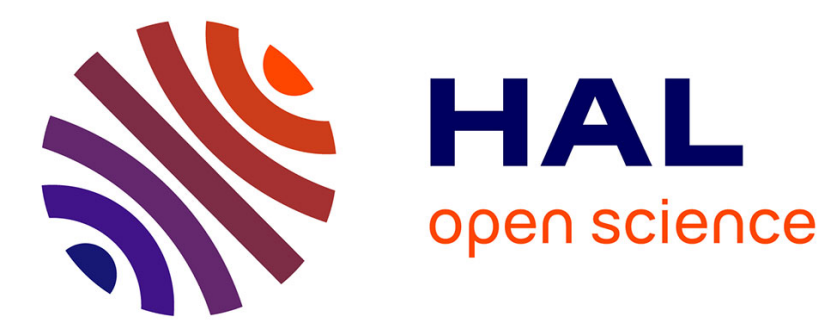

\title{
La coutume, la CNUDM et la Cour internationale de Justice
}

\author{
Guillaume Le Floch
}

\section{To cite this version:}

Guillaume Le Floch. La coutume, la CNUDM et la Cour internationale de Justice. Angela Del Vecchio; Roberto Virzo. Interpretations of the United Nations Convention on the Law of the Sea by International Courts and Tribunals, Springer, pp.41-57, 2019, 978-3-030-10773-4. 10.1007/978-3-03010773-4_3. hal-02169648

\section{HAL Id: hal-02169648 https://hal-univ-rennes1.archives-ouvertes.fr/hal-02169648}

Submitted on 1 Jul 2019

HAL is a multi-disciplinary open access archive for the deposit and dissemination of scientific research documents, whether they are published or not. The documents may come from teaching and research institutions in France or abroad, or from public or private research centers.
L'archive ouverte pluridisciplinaire HAL, est destinée au dépôt et à la diffusion de documents scientifiques de niveau recherche, publiés ou non, émanant des établissements d'enseignement et de recherche français ou étrangers, des laboratoires publics ou privés. 


\section{LA COUTUME, LA CNUDM ET LA COUR INTERNATIONALE DE JUSTICE Guillaume Le Floch Professeur à l'Université de Rennes (IDPSP)}

Durant des siècles, le droit de la mer a pour ainsi dire exclusivement reposé sur la coutume $^{1}$. Ce n'est qu'au lendemain de la seconde guerre mondiale ${ }^{2}$ que celui-ci, à l'image d'autres branches du droit international, fit l'objet d'une vaste entreprise de codification à trois reprises ${ }^{3}$. Si la deuxième conférence des Nations Unies sur le droit de la mer se solda par un échec, les deux autres en revanche furent une réussite. La première donna lieu à l'adoption de quatre conventions à Genève en $1958^{4}$ tandis que la troisième s'acheva par l'adoption d'un texte monumental : la Convention des Nations Unies sur le droit de la mer (CNUDM). Destiné à établir un ordre juridique pour les mers et les océans ${ }^{5}$, la convention de Montego Bay mérite indiscutablement le nom de «Constitution des océans » comme l'a qualifiée le président Tommy $\mathrm{Koh}^{6}$.

En dépit de son importance indiscutable, la Convention des Nations Unies sur le droit de la mer n’a pas conduit à la disparition de la coutume en ce domaine. Ainsi que le précise expressément le dernier paragraphe du préambule de la "Constitution des océans ", "les questions qui ne sont pas réglementées par la Convention continueront d'être régies par les règles et principes du droit international général », ce qui inclut des domaines importants du droit de la mer à l'instar notamment du droit des conflits armés en mer, du droit de la neutralité, des questions relatives aux baies et titres historiques, à l'accès au port ou bien encore à la compétence des navires dans le port ${ }^{7}$. La CNUDM ne signe donc pas l'acte de décès de la coutume. Au contraire même, loin de l'éradiquer, elle a contribué à sa revitalisation.

Comme l'a longuement et soigneusement examiné la Cour internationale de Justice (CIJ) dans son arrêt sur le Plateau continental de la mer du Nord, les traités de codification sont susceptibles de produire trois types d'effet sur les normes coutumières. Certaines dispositions se bornent à transcrire sous forme conventionnelle une règle coutumière existante et ont alors un effet déclaratoire. D’autres ont pour effet de cristalliser une coutume naissante en ce sens que le processus de maturation de la règle coutumière est mené à termes à travers l'élaboration, la négociation et l'adoption du traité de codification. Certaines dispositions enfin ont un caractère foncièrement innovateur mais sont susceptibles ultérieurement de connaître une mutation coutumière ${ }^{8}$. Quel que soit le cas de figure, le résultat est le même : la

\footnotetext{
${ }^{1}$ Colombos pouvait ainsi écrire en 1952 que « la coutume forme la source la plus importante du droit de la mer » : Constantine John Colombos, Le droit international de la mer, Paris, Pedone, 1952, 1.

${ }^{2}$ Il faut toutefois ici signaler l'échec de la Conférence pour la codification du droit international qui se déroula du 13 mars au 2 avril 1930 à La Haye.

3 Sur la question : Franck Latty, Du droit coutumier aux premières tentatives de codification, in M. Forteau, J.M. Thouvenin (dirs.), Traité de droit international de la mer, Paris, Pedone, 2017, 35-54.

${ }^{4}$ Ont de la sorte été adoptées le 29 avril 1958 : la Convention sur la mer territoriale et la zone contiguë, la Convention sur la haute mer, la Convention sur la pêche et la conservation des ressources biologiques de la haute mer et la Convention sur le plateau continental.

${ }^{5} 4$ elinéa du Préambule.

6 "A Constitution for the Oceans ", Remarks by T.B. Koh, 1982 <http://www.un.org/depts/los/convention_agreements/texts/koh_english.pdf>.

${ }^{7}$ V. Michael Wood, Le rôle contemporain du droit international coutumier, in M. Forteau, J.-M. Thouvenin (dirs.), Traité de droit international de la mer, Paris, Pedone, 2017, 68-77, 74.

${ }^{8}$ CIJ, Plateau continental de la mer du Nord (Allemagne c. Danemark/Pays-Bas), arrêt, 20 Fév. 1969, § 60-82. Sur ce processus : Georges Abi-Saab, La coutume dans tous ses états ou le dilemme du développement du droit
} 
norme en cause se retrouve à avoir un fondement tout à la fois conventionnel et coutumier. Cette caractéristique est loin d'être négligeable dès lors que la convention de codification ne fait pas l'objet d'une ratification universelle. Ce qui est le cas de la Convention des Nations Unies sur le droit de la mer dont le nombre de parties s'élève à 168. S'il s'agit d'un nombre conséquent, il n'en demeure pas moins que certains Etats dotés d'importantes façades maritimes manquent encore et toujours à l'appel à l'instar de la Colombie, des Etats-Unis, de l'Iran, d'Israël, du Pérou, de la Turquie ou bien encore du Venezuela. Etant tiers à la Convention, ces Etats ne sauraient se prévaloir de la CNUDM sauf à ce que les dispositions invoquées aient également un fondement coutumier ${ }^{9}$. C'est une question à laquelle a été confrontée la Cour internationale de Justice à de nombreuses reprises.

Le droit de la mer occupe une place prépondérante dans la jurisprudence de la $\mathrm{CIJ}^{10}$. Cependant, aussi surprenant que cela puisse paraître, la Cour n'a été somme toute que peu souvent confrontée à l'application de la CNUDM. Certains différends, à l'image de celui opposant l'Australie au Japon dans l'affaire de la Chasse à la baleine, ont été réglés sur le fondement d'un autre traité ${ }^{11}$. D'autres, de manière plus fréquente, l'ont été sur la base du droit coutumier dans la mesure où la CNUDM n'était pas applicable : soit parce qu'elle n'était pas entrée en vigueur pour les affaires antérieures à $1994^{12}$, soit, pour les affaires postérieures, parce que l'une au moins des deux parties au différend ne l'avait pas ratifiée ${ }^{13}$. Il en résulte que le premier arrêt de la Cour qui fasse application de la CNUDM est celui de la Frontière terrestre et maritime entre le Cameroun et le Nigéria rendu le 10 octobre 2002, soit près de 20 après l'adoption de la Convention ${ }^{14}$. Elle a depuis lors eu également l'occasion de l'appliquer dans ses arrêts rendus respectivement le 8 octobre 2007 dans l'affaire du Différend territorial et maritime entre le Nicaragua et le Honduras dans la mer des Caraïbes ${ }^{15}$, le 3

international général dans un monde éclaté, in Etudes en l'honneur de Roberto Ago, Milan, Guiffré, 1987, 4 vol., t. 1, 53-65, 53.

${ }^{9}$ Une norme coutumière universelle est opposable à tous les Etats à l'exception néanmoins de ceux qui y ont objecté de manière persistante. C'est le cas par exemple de la Turquie qui s'est opposée et continue aujourd'hui encore de le faire à l'extension de la mer territoriale à 12 milles nautiques. V. James A. Green, The Persistent Objector Rule in International Law, Oxford, OUP, 2016, 53.

${ }^{10}$ V. notamment : Vaughan Lowe, Antonios Tzanakopoulos, The Development of the Law of the Sea by the International Court of Justice, in C.J. Tams, J. Sloan (ed.), The Development of International Law by the International Court of Justice, Oxford, OUP, 2013, 177-193.

${ }^{11}$ En l'espèce, il s'agissait de la Convention internationale pour la réglementation de la chasse à la baleine de 1946: CIJ, Chasse à la baleine dans l'Antarctique (Australie c. Japon ; Nouvelle-Zélande (intervenant)), 31 Mars 2014.

12 V. CIJ, Délimitation de la frontière maritime dans la région du golfe du Maine (Canada/Etats-Unis d'Amérique), Arrêt, 12 Oct. 1984 ; CIJ, Plateau continental (Jamahiriya arabe libyenne/Malte), Arrêt, 3 Juin 1985 ; CIJ, Différend frontalier terrestre, insulaire et maritime (El Salvador/Honduras ; Nicaragua (intervenant)), Arrêt, 11 Sept. 1992 ; CIJ, Délimitation maritime dans la région située entre le Groenland et Jan Mayen (Danemark c. Norvège), Arrêt, 14 Juin 1993.

${ }^{13}$ V. CIJ, Délimitation maritime et questions territoriales entre Qatar et Bahrë̈n (Qatar c. Bahrë̈n), Arrêt, 16 Mars 2001, § 167 ; CIJ, Différend territorial et maritime (Nicaragua c. Colombie), Arrêt, 19 Nov. 2012, § 114 ; CIJ, Différend maritime (Pérou c. Chili), Arrêt, 27 Janv. 2014, § 178.

${ }^{14}$ CIJ, Frontière terrestre et maritime entre le Cameroun et le Nigéria (Cameroun c. Nigéria ; Guinée Equatoriale (intervenant)), Arrêt, 10 Oct. 2002, § 285. La CNUDM a été ratifiée par le Cameroun en 1985 et par le Nigéria en 1986. Le Cameroun a saisi la Cour par requête unilatérale le 29 mars 1994 soit quelques mois avant l'entrée en vigueur de la Convention (16 novembre 1994). La Convention est dès lors devenue applicable au fond du litige dont le caractère était continu.

${ }^{15}$ CIJ, Différend territorial et maritime entre le Nicaragua et le Honduras dans la mer des Caraïbes (Nicaragua c. Honduras), Arrêt, 8 Oct. 2007, § 261. La CNUDM a été ratifiée par le Honduras en 1993 tandis qu'elle l’a été en 2000 par le Nicaragua alors même que l’instance était pendante. 
février 2009 dans l'affaire de la Délimitation maritime en mer Noire ${ }^{16}$, le 2 février 2017 dans l'affaire de la Délimitation maritime dans l'Océan indien ${ }^{17}$ et le 2 février 2018 dans l'affaire de la Délimitation maritime dans la mer des Caraïbes et l'Océan Pacifique ${ }^{18}$. Toutes les autres affaires de délimitation maritime, en revanche, ont été rendues sur le fondement du droit coutumier. Cette situation contraste singulièrement avec celle du Tribunal international du droit de la mer (TIDM) qui a systématiquement été amené à trancher des différends se rapportant à l'interprétation ou à l'application de la Convention des Nations Unies sur le droit de la mer. Cette différence s'explique par la compétence plus étendue dont bénéficie la Cour. Contrairement à elle, en effet, le Tribunal ne peut être saisi que par les parties à la CNUDM ${ }^{19}$ et pour des différends se rapportant à cette convention.

Si la Cour a ainsi été amenée, pour la première fois, à faire application de la Convention des Nations Unies sur le droit de la mer vingt ans après son adoption, elle n'a pas pour autant attendu tout ce temps avant de l'interpréter. Elle a pu en tenir compte avant même qu'elle n'entre en vigueur voire avant qu'elle ne soit complètement finalisée.

Dans l'affaire du Plateau continental (Tunisie/Libye), elle avait précisé qu'elle « ne saurait (...) négliger une disposition du projet de convention si elle venait à conclure que sa substance lie tous les membres de la communauté internationale du fait qu'elle consacre ou cristallise une règle de droit coutumier préexistante ou en voie de formation ${ }^{20}$. Elle avait en outre accepté de donner suite au compromis conclu en 1977 entre les parties au différend qui l'invitait à statuer en tenant compte «des nouvelles tendances admises à la troisième conférence sur le droit de la mer ${ }^{21}$. Ce qui avait du reste fait couler beaucoup d'encre à l'époque au sein et en dehors du prétoire ${ }^{22}$. Dans l'affaire du Plateau continental (Libye/Malte), elle avait fait observer que la CNUDM : «ayant été adoptée par l'écrasante majorité des Etats, (...) revêt une importance majeure, de sorte que, même si les Parties ne l'invoquent pas, il incombe manifestement à la Cour d'examiner jusqu'à quel point l'une quelconque de ses dispositions pertinentes lie les Parties en tant que règle de droit international coutumier ${ }^{23}$.

La Convention des Nations Unies sur le droit de la mer a eu une influence certaine sur la coutume. Si plusieurs de ses dispositions ne font que codifier des normes coutumières existantes d'autres les ont cristallisées voire ont donné naissance à de nouvelles. Au gré de sa jurisprudence, la Cour internationale de Justice a été amenée à constater que plusieurs des

\footnotetext{
${ }^{16}$ CIJ, Délimitation maritime en mer Noire (Roumanie c. Ukraine), Arrêt, 3 Fév. 2009, § 31. La Roumanie a déposé son instrument de ratification le 17 décembre 1996 et l’Ukraine le 26 juillet 1999.

17 CIJ, Délimitation maritime dans l'Océan indien (Somalie c. Kenya), exceptions préliminaires, Arrêt, 2 Fév. 2017, § 16. Le Kenya et la Somalie ont ratifié la CNUDM en 1989. La Cour aura donc l’occasion d'appliquer la Convention lorsqu'elle rendra son arrêt au fond.

${ }^{18}$ CIJ, Délimitation maritime dans la mer des Caraïbes et l'océan Pacifique (Costa Rica c. Nicaragua), Arrêt, 2 Fév. 2018, § 90. Le Costa Rica a ratifié la CNUDM en 1992 et le Nicaragua en 2000. Dans son arrêt, la Cour n’a pas fait la moindre référence au droit coutumier. Enfin, il faut préciser que dans l'affaire de la Souveraineté sur Pedra Branca/Pulau Batu Puteh, Middle Rocks et South Ledge (Malaisie/Singapour), la Cour s'est référée à l'article 13 de la CNUDM : CIJ, Arrêt, 23 Mai 2008, § 292. Singapour et la Malaisie sont respectivement parties à la Convention depuis 1994 et 1996.

${ }^{19}$ Exceptée l'hypothèse où le TIDM est saisi en application de l'article 282 de la CNUDM. Sur la compétence du TIDM v. notamment : V. Boré Eveno, Les Etats et le Tribunal international du droit de la mer, in G. Le Floch (dir.), Les vingt ans du Tribunal international du droit de la mer, Paris, Pedone, 2018 (à paraître).

${ }^{20}$ CIJ, Plateau continental (Tunisie/Jamahiriya arabe libyenne), Arrêt, 24 Fév. 1982, § 24.

21 Article 1 du compromis d'arbitrage signé le 10 juin 1977 à Tunis: CIJ, Plateau continental (Tunisie/Jamahiriya arabe libyenne), Arrêt, 24 Fév. 1982, § 2.

${ }^{22}$ V. Emmanuel Decaux, Les eaux mêlées de l'arbitrage et de la justice : droit de la mer et règlement des différends, in La Mer et son Droit: Mélanges offerts à Laurent Lucchini et Jean-Pierre Quéneudec, Paris, Pedone, 2003, 159-176, 168.

${ }^{23}$ CIJ, Plateau continental (Jamahiriya arabe libyenne/Malte), Arrêt, 3 Juin 1985, § 27.
} 
dispositions de la CNUDM reflètent le droit coutumier. Après en avoir dressé la liste (1), il conviendra de revenir sur les méthodes d’identification utilisées par la Cour (2).

\section{Recension des dispositions de la CNUDM qualifiées de coutumières par la Cour internationale de Justice}

La CNUDM est une convention de codification. Il en résulte que plusieurs de ses dispositions ont également un fondement coutumier. En 1982, les Etats-Unis, qui ne voulaient pas ratifier la Convention, prétendirent que toutes ses dispositions reflétaient la coutume à l'exception de celles relatives à la partie $\mathrm{XI}^{24}$. Cette position était bien entendu excessive, ne serait-ce seulement que parce que les dispositions institutionnelles ne peuvent acquérir le rang de normes coutumières ${ }^{25}$. Ce ne sont pas, pour reprendre l'expression de la Cour internationale de Justice, des dispositions ayant un «caractère fondamentalement normatif $»^{26}$. Il est donc nécessaire d'examiner une à une chaque disposition pour établir si oui ou non la norme qu'elle renferme a un équivalent dans le droit coutumier.

A travers sa jurisprudence, la Cour internationale de Justice a été amenée à reconnaître un caractère coutumier à plusieurs dispositions de la CNUDM. Il en est allé de la sorte des articles 2, paragraphe 1 sur le régime juridique de la mer territoriale ${ }^{27}, 3$ sur la largeur de la mer territoriale ${ }^{28}, 5$ sur les lignes de base normale ${ }^{29}, 7$, paragraphe 4 sur les lignes de bases droites $^{30}, 10$ sur les baies ${ }^{31}, 13$ sur les hauts fonds découvrant ${ }^{32}, 15$ sur la délimitation de la mer territoriale des Etats dont les côtes sont adjacentes ou se font face ${ }^{33}$, 17 sur le droit de passage inoffensif dans la mer territoriale ${ }^{34}, 18$ sur la signification du terme passage ${ }^{35}, 57$ sur la largeur de la Zone économique exclusive $(\mathrm{ZEE})^{36}$, 58 sur la liberté de navigation dans la

\footnotetext{
${ }^{24}$ V. James L. Malone, The United States and the Law of the Sea after UNCLOS III, in Law and Contemporary Problems, 1983, 29-36, spec. 29, 33. Pour une critique de cette position : William T. Burke, Customary Law of the Sea : Advocacy or Disinterested Scholarship, in Yale Journal of International Law, 1989, 508-527.

${ }^{25}$ Robin Churchill, The 1982 United Nations Convention on the Law of the Sea, in D. Rothwell et al. (eds.), The Oxford Handbook of the Law of the Sea, Oxford, OUP, 2016, 24-45, 37-38. V. également : Tullio Treves, UNCLOS at Thirty: Open Challenges, Ocean Yearbook, 2013, 49-66, 51.

${ }^{26}$ Plateau continental de la mer du Nord (République fédérale d'Allemagne/Danemark; République fédérale d'Allemagne/Pays-Bas), Arrêt, 20 Fév. 1969, § 72.

27 CIJ, Activités militaires et paramilitaires au Nicaragua et contre celui-ci (Nicaragua c. Etats-Unis d’Amérique), Arrêt, 27 Juin 1986, § 212. V. également de manière implicite : CIJ, Délimitation maritime et questions territoriales entre Qatar et Bahreïn (Qatar c. Bahrë̈n), Arrêt, 16 Mars 2001, § 204 ; CIJ, Différend territorial et maritime (Nicaragua c. Colombie), Arrêt, 19 Nov. 2012, § 26.

${ }^{28}$ CIJ, Différend territorial et maritime (Nicaragua c. Colombie), Arrêt, 19 Nov. 2012, § 177.

${ }^{29}$ CIJ, Délimitation maritime et questions territoriales entre Qatar et Bahreïn (Qatar c. Bahrë̈n), Arrêt, 16 Mars 2001, § 184.

${ }^{30}$ Ibid., § 201.

${ }^{31}$ CIJ, Différend frontalier terrestre, insulaire et maritime (El Salvador/Honduras ; Nicaragua (intervenant)), Arrêt, 11 Sept. 1992, § 383.

${ }^{32}$ CIJ, Délimitation maritime et questions territoriales entre Qatar et Bahrë̈n (Qatar c. Bahrë̈n), Arrêt, 16 Mars 2001, § 201 (elle se limite toutefois au paragraphe 1 de l'article 13) ; CIJ, Différend territorial et maritime (Nicaragua c. Colombie), Arrêt, 19 Nov. 2012, § 182 (elle englobe les deux paragraphes de l'article 13).

${ }^{33}$ CIJ, Délimitation maritime et questions territoriales entre Qatar et Bahreïn (Qatar c. Bahreïn), Arrêt, 16 Mars 2001, § 175-176.

${ }^{34}$ Ibid., § 223. Elle ne fait cependant pas mention de l'article 17. Elle se contente de signaler que le droit de passage inoffensif dans la mer territoriale est consacré par le droit international coutumier. V. infra note 44.

35 CIJ, Activités militaires et paramilitaires au Nicaragua et contre celui-ci (Nicaragua c. Etats-Unis d’Amérique), Arrêt, 27 Juin 1986, § 214.

${ }^{36}$ CIJ, Plateau continental (Jamahiriya arabe libyenne/Malte), Arrêt, 3 Juin 1985, § 34.
} 
$\mathrm{ZEE}^{37}, 74$, paragraphe 1 sur la délimitation de la ZEE des Etats dont les côtes sont adjacentes ou se font face $e^{38}, 76$, paragraphe 1 sur la définition du plateau continental ${ }^{39}, 83$, paragraphe 1 sur la délimitation du plateau continental ${ }^{40}, 87$, paragraphe 1 a) sur la liberté de navigation en haute mer $^{41}$ et 121 sur le régime juridique des îles ${ }^{42}$.

Plusieurs des dispositions cardinales de la CNUDM se sont par conséquent vues reconnaitre un caractère coutumier par la CIJ. Dans la très grande majorité des cas, la Cour l'a reconnu de manière explicite. Dans l'affaire du Différend territorial et maritime (Nicaragua c. Colombie), par exemple, elle affirme sans ambiguïté aucune que « la définition du plateau continental énoncée au paragraphe 1 de l'article 76 de la CNUDM fait partie du droit international coutumier ${ }^{43}$. Dans certains cas, néanmoins, cette reconnaissance est moins affirmée. Il peut en effet lui arriver de considérer qu'une règle consacrée dans la CNUDM reflète le droit coutumier sans toutefois viser expressément la Convention de Montego Bay. Dans l'affaire de la Délimitation maritime et questions territoriales entre Qatar et Bahreïn, la Cour a rappelé le caractère coutumier du droit de passage inoffensif dans la mer territoriale sans faire mention des dispositions pertinentes de la CNUDM ${ }^{44}$. Il peut également lui arriver de laisser à l'interprète le soin de le déduire ${ }^{45}$. A titre d'illustration, dans l'affaire précitée, elle affirme «que, selon les règles de droit international applicables, la ligne de base normale à partir de laquelle est mesurée la largeur de la mer territoriale est la laisse de basse mer le long de la côte (article 5 de la convention de 1982 sur le droit de la mer) » ${ }^{46}$. La Cour ne dit donc pas expressément que l'article 5 est une disposition qui reflète le droit coutumier. Il est néanmoins possible de le déduire : à partir du moment où les parties à l'instance ne sont pas liées à la Convention et que la Cour leur applique la norme, c'est que celle-ci a nécessairement un fondement coutumier.

37 CIJ, Activités militaires et paramilitaires au Nicaragua et contre celui-ci (Nicaragua c. Etats-Unis d'Amérique), Arrêt, 27 juin 1986, § 214.

${ }^{38}$ CIJ, Délimitation maritime et questions territoriales entre Qatar et Bahrë̈n (Qatar c. Bahreïn), Arrêt, 16 Mars 2001, § 167 et s. ; CIJ, Différend territorial et maritime (Nicaragua c. Colombie), Arrêt, 19 Nov. 2012, § 139 et s. ; CIJ, Différend territorial et maritime (Nicaragua c. Colombie), Arrêt, 19 Nov. 2012, § 139 ; Différend maritime (Pérou c. Chili), Arrêt, 27 Janv. 2014, § 179.

${ }^{39}$ CIJ, Plateau continental (Jamahiriya arabe libyenne/Malte), Arrêt, 3 Juin 1985, § 77 ; CIJ, Différend territorial et maritime (Nicaragua c. Colombie), Arrêt, 19 Nov. 2012, § 118.

${ }^{40}$ CIJ, Plateau continental (Jamahiriya arabe libyenne/Malte), Arrêt, 3 Juin 1985, § 77 ; CIJ, Différend territorial et maritime (Nicaragua c. Colombie), Arrêt, 19 Nov. 2012, § 167 et s. ; CIJ, Différend territorial et maritime (Nicaragua c. Colombie), Arrêt, 19 Nov. 2012, § 139 ; CIJ, Différend maritime (Pérou c. Chili), Arrêt, 27 Janv. 2014, § 179.

${ }^{41}$ CIJ, Activités militaires et paramilitaires au Nicaragua et contre celui-ci (Nicaragua c. Etats-Unis d’Amérique), Arrêt, 27 Juin 1986, § 214.

42 CIJ, Différend territorial et maritime (Nicaragua c. Colombie), Arrêt, 19 Nov. 2012, § 139. Dans l'affaire Qatar c. Bahrë̈n, elle n'avait reconnu le caractère coutumier que des deux premiers paragraphes: CIJ, Délimitation maritime et questions territoriales entre Qatar et Bahreïn (Qatar c. Bahreïn), Arrêt, 16 Mars 2001, $\S 195$ (paragraphe 1) et $\S 185$ (paragraphe 2).

${ }^{43}$ CIJ, Différend territorial et maritime (Nicaragua c. Colombie), Arrêt, 19 Nov. 2012, § 118.

44 « Les eaux s'étendant entre les îles Hawar et les autres îles bahreïnites constituent, non des eaux intérieures de Bahreïn, mais la mer territoriale de cet Etat. Par voie de conséquence, les navires de Qatar, comme d'ailleurs ceux de tous les autres Etats, jouissent dans ces eaux du droit de passage inoffensif consacré par le droit international coutumier. De même, les navires de Bahreïn, comme d'ailleurs ceux de tous les autres Etats, jouissent dans la mer territoriale de Qatar de ce droit de passage inoffensif »(italiques ajoutés) : CIJ, Délimitation maritime et questions territoriales entre Qatar et Bahreïn (Qatar c. Bahreïn), Arrêt, 16 Mars 2001, $\S 223$.

${ }^{45}$ V. notamment : Brian McGarry, The Development of Custom in Territorial Dispute Settlement, in Journal of International Dispute Settlement, 2017, 339-365, 343.

${ }^{46}$ CIJ, Délimitation maritime et questions territoriales entre Qatar et Bahrë̈n (Qatar c. Bahrë̈n), Arrêt, 16 Mars 2001, § 184. 
Bien entendu, la CIJ n'a pas été conduite à se prononcer sur l'ensemble des dispositions de la Convention. Or, plusieurs d'entre-elles sont considérées comme reflétant indiscutablement le droit coutumier. C'est le cas par exemple de l'article 19 sur la signification de l'expression passage inoffensif ${ }^{47}$ ou bien encore de l'article 97 sur la juridiction pénale exclusive en matière d'abordage en haute mer ${ }^{48}$. D'autres, en revanche, soulèvent certaines interrogations. Il en va par exemple de celles qui sont tout à la fois très détaillées et très spécifiques à l'instar des dispositions relatives au statut d'Etat archipélagique. Dans l'affaire de la Délimitation maritime et questions territoriales entre Qatar et Bahrë̈n, les parties avaient émis des vues divergentes sur cette question. Contrairement au Qatar, Bahreïn prétendait que la partie IV de la Convention reflétait le droit international coutumier. Par souci d'économie judiciaire, la Cour n’a toutefois pas jugé utile de se prononcer sur cette question ${ }^{49}$. Au regard de la pratique des Etats ainsi que de la large acceptation des lignes de base archipélagiques, il est sans doute possible aujourd'hui d'affirmer que ces dispositions ont un caractère coutumier. Comme le mentionne Sean D. Murphy, “[m]any States Parties to the LOS Convention have taken advantage of Part IV : Antigua and Barbuda ; Bahamas ; Cape Verde ; Comoros ; Fiji ; Grenada ; Indonesia ; Jamaica : Kiribati ; Maldives ; Marshall Islands ; Mauritius ; Papua New Guinea ; Philippines ; Saint Vincent and the Grenadines ; São Tomé and Príncipe ; Seychelles ; Solomon Islands ; Trinidad and Tobago ; Tuvalu ; and Vanuatu. Given such practice, and the general acceptance of the archipelagic baselines if done consistently with Part IV by States that are not parties to the LOS Convention, it seems likely that the rules set forth in Part IV now reflect customary international law" ${ }^{50}$. La question peut également se poser à l'égard de bien d'autres dispositions comme celle relative au passage dans les détroits ${ }^{51}$.

Entre 1982 et aujourd'hui, le nombre de normes bénéficiant d'un statut tout à la fois conventionnel et coutumier n'a cessé de croître compte tenu des interactions constantes entre la CNUDM (convention de codification) et la coutume. Ainsi que le fait remarquer Tullio Treves, "[a]t present it can be said that there is a presumption that the provisions of the Convention correspond to customary law. It is, however, a rebuttable presumption as, again

\footnotetext{
${ }^{47}$ V. en ce sens : Robin Rolf Churchill, Alan Vaughan Lowe, The Law of the Sea, Manchester, MUP, $3^{\text {rd }}$ ed., $1999,87$.

${ }^{48}$ David Anderson, Modern Law of the Sea: Selected Essays, Leiden/Boston, Martinus Nijhoff, 2008, 268. Il en va de même des articles 95, 96 et 236 : Donald R. Rothwell, Tim Stephens, The International Law of the Sea, Oxford, Hart, 2010, 161.

${ }^{49}$ CIJ, Délimitation maritime et questions territoriales entre Qatar et Bahrë̈n (Qatar c. Bahrë̈), Arrêt, 16 Mars 2001, § 181-183. D’après Alberto Alvarez-Jimenez, “[a]lthough the Court did not explicitly declare that Part IV of the Convention reflected customary international law, it is important to mention that the Court implicitly regarded it to be so, since the Court assessed the provision by examining the condition for its application and determined that Bahrain did not meet it. The existence of the implicit recognition of customary norms in this case is also supported by the fact that the Court did not make use of the arguendo technique, which exists in international adjudication. According to this technique, the Court could have inverted the order of analysis by stating that, before addressing the customary character, it would assume, arguendo, that Part IV was a customary rule and proceed to assess first whether Bahrain met the requirements of archipelagic States provided for therein. Once the Court found that Bahrain did not meet them, the Court could have stated that there was no need to rule": Methods for the identification of customary international law in the International Court of Justice's jurisprudence: 2000-2009, in ICLQ, 2011, 681-712, 701-702.

${ }^{50}$ Sean D. Murphy, International Law Relating to Islands, in RCADI, Vol. 386, 9-265, 148. V. également : Robin Rolf Churchill, Alan Vaughan Lowe, The Law of the Sea, Manchester, MUP, $3^{\text {rd }}$ ed., 1999, 130 ; Donald R. Rothwell, Tim Stephens, The International Law of the Sea, Oxford, Hart, 2010, 189. Pour une position plus nuancée : Yoshifumi Tanaka, The International Law of the Sea, Cambridge, Cambridge University Press, 2012, 109.

${ }^{51}$ Hugo Caminos, Vincent Cogliati-Bantz, The Legal Regime of Straits. Contemporary Challenges and Solutions, Cambridge, CUP, 2014, 452-471.
} 
on a case-by-case basis, evidence can be submitted to argue that a specific provision has a merely treaty character" ${ }^{32}$. Il reste dès lors à s'interroger sur la méthode d'identification utilisée par la Cour.

\section{Une méthode d'identification parfois contestable}

Comme l'a clairement rappelé la CIJ à maintes reprises, l'existence d'une norme coutumière est attestée par la réunion cumulative et convergente de deux éléments : la pratique et l'opinio juris ${ }^{53}$. La réunion de ces deux éléments est une condition à la fois nécessaire et suffisante pour pouvoir dégager une coutume. En théorie, chacun des deux éléments doit faire l'objet d'une étude distincte préalable. En pratique, cependant, il en va souvent autrement ${ }^{54}$.

Dans le domaine du droit de la mer, à l'exception notable de l'affaire du Plateau continental de la mer du Nord, la Cour ne s'est jamais livrée à une quelconque étude approfondie de la pratique et de l'opinio juris ${ }^{55}$. Elle se borne en effet à chaque fois à constater que telle ou telle disposition de la convention de Montego Bay a un caractère coutumier sans fournir la moindre explication ${ }^{56}$. A titre d'illustration, dans l'affaire de la Délimitation maritime et questions territoriales entre Qatar et Bahrë̈n, elle s'est contentée d'affirmer que l'article 13 paragraphe 1 de la CNUDM sur les hauts-fonds découvrants reflétait « le droit international coutumier ». La seule justification qu'elle a avancée au soutien de son affirmation est que cette disposition figurait également à l'article 11 paragraphe 1 de la Convention de 1958 sur la mer territoriale et la zone contiguë ${ }^{57}$. De même, dans l'affaire du Différend territorial et maritime (Nicaragua c. Colombie) ${ }^{58}$, elle s'est bornée à rappeler

\footnotetext{
52 Tullio Treves, UNCLOS at Thirty: Open Challenges, Ocean Yearbook, 2013, 49-66, 51. V. également Michael Wood, Le rôle contemporain du droit international coutumier, in M. Forteau, J.-M. Thouvenin (dirs.), Traité de droit international de la mer, Paris, Pedone, 2017, 68-77, 70. Comme le relève Vaughan Lowe, «[w]hile that process of translation into customary international law may have fostered a general willingness to sign and then ratify the Convention, it may also have had a paradoxically opposite effect. Because rights tend to pass into customary international law more easily than obligations, most of the benefits of the 1982 Convention can now be claimed as rights under customary law, and many of the obligations - notably the dispute settlement obligations under Part XV of the Convention - can be avoided by refraining from ratifications ": Was it Worth the Effort?, in IJMCL, 2012, 875-881, 879.

${ }^{53}$ V. par exemple : CIJ, Plateau continental de la mer du Nord (Allemagne c. Danemark/Pays-Bas), Arrêt, 20 Fév. 1969, § 77.

${ }^{54}$ V. notamment Peter Haggenmacher, La doctrine des deux éléments du droit coutumier dans la pratique de la Cour Internationale, in RGDIP, 1986, 5-126.

55 J. Ashley Roach, Today's Customary International Law of the Sea, in ODIL, 2014, 239-259, 242. V. aussi Tullio Treves, Customary International Law, in Max Planck Encyclopaedia of Public International Law, 2006, § 73-75.

${ }^{56}$ Cela n'est cependant pas propre au droit de la mer. V. en sens : Stephan Talmon, Determining Customary International Law : The ICJ's Methodology between Induction, Deduction and Assertion, in EJIL, 417-443, passim. 437-438. De la même façon, le TIDM «s'est abstenu de rentrer dans des considérations liées à la détermination de l'existence ou du contenu d'une règle de droit international coutumier en analysant la pratique et l'opinio juris pertinentes. Il a préféré, au moins dans la plupart des affaires, s'en remettre à l'autorité de la CIJ et de la Commission du droit international »: Le Tribunal international du droit de la mer et le droit international coutumier, in L. Lijnzaad (dir.), Le juge et la coutume internationale, Leiden/Boston, Brill/Nijhoff, 2016, 25-45, 45.

${ }^{57}$ CIJ, Délimitation maritime et questions territoriales entre Qatar et Bahrë̈n (Qatar c. Bahreïn), Arrêt, 16 Mars 2001, § 201.

${ }^{58}$ CIJ, Différend territorial et maritime (Nicaragua c. Colombie), Arrêt, 19 Nov. 2012, § 182.
} 
qu'elle avait déjà reconnu un caractère coutumier à cette disposition tout en y ajoutant le deuxième paragraphe ${ }^{59}$. Cette façon de faire est susceptible de trouver des justifications.

En premier lieu, il est tout à fait possible de considérer que la large participation des Etats à la Convention de Montego Bay peut être perçue comme génératrice d'une pratique substantielle $^{60}$. En deuxième lieu, les règles de la Convention, partie XI bien entendu exceptée, ne font pas l'objet d'oppositions significatives de la part des Etats. En troisième et dernier lieu, comme l'a du reste souvent mis en exergue la Cour, certaines dispositions figuraient déjà dans les Conventions de Genève de 1958. Par ailleurs, lorsqu'elle a déjà reconnu un caractère coutumier à une norme, il n'est pas nécessaire qu'elle s'emploie de nouveau à démontrer l'existence de la pratique et de l'opinio juris.

Il n'en demeure pas moins que dans certaines hypothèses, cette attitude n'est pas sans susciter des critiques. C'est en particulier le cas lorsque la disposition dont il s'agit est controversée à l'image du troisième paragraphe de l'article 121 de la Convention ${ }^{61}$. Dans l'affaire de la Délimitation maritime et questions territoriales entre Qatar et Bahreïn, la Cour avait reconnu un caractère coutumier aux deux premiers paragraphes de cette disposition ${ }^{62}$. Elle ne s'était en revanche pas prononcée sur le troisième paragraphe aux termes duquel « [l]es rochers qui ne se prêtent pas à l'habitation humaine ou à une vie économique propre n'ont pas de zone économique exclusive ni de plateau continental ». Plusieurs auteurs considéraient que cette disposition ne reflétait pas le droit coutumier ${ }^{63}$ dans la mesure où, non seulement la convention de Genève sur le plateau continental ne contenait pas une telle exception mais en outre la pratique atteste que plusieurs Etats ont pu revendiquer une ZEE et un plateau continental pour des espaces relevant indiscutablement de la catégorie des « rochers ${ }^{64}$. D’autres pour des raisons inverses défendaient le caractère coutumier de cette

\footnotetext{
${ }^{59}$ CIJ, Délimitation maritime et questions territoriales entre Qatar et Bahrë̈n (Qatar c. Bahrë̈n), Arrêt, 16 Mars 2001, § 201 ; CIJ, Différend territorial et maritime (Nicaragua c. Colombie), arrêt, 19 Nov. 2012, § 182 (où elle englobe les deux paragraphes).

${ }^{60}$ D'aucuns ont toutefois prétendu que la Convention ne pouvait exercer une quelconque influence dans la mesure où elle avait été adoptée dans le cadre d'un package deal. "If one assumes that the package deal was solidified at the time that the Convention was formally adopted, then those of its provisions that had not attained customary status by that date may have been precluded from ever doing so": Hugo Caminos, Michael R. Molitor, Progressive Development of International Law and the Package Deal, in American Journal of International Law, 1985, 871-890, 888. Au soutien de leur argumentation, ces auteurs se réfèrent à différentes déclarations faites par des participants à la troisième conférence des Nations Unies sur le droit de la mer : ibid., 877. Cette affirmation mérite néanmoins d’être remise en cause. Comme le precise Stephen Vasciannie, “[t]his approach is clearly untenable: by requiring States to choose between a tabula rasa and the entire Convention, it would create considerable havoc in the international legal order. Not only that, it would also require States to deny the independent status of custom as a source of obligations in matters falling within the purview of the LOSC: as this requirement has no basis in law, it cannot be supported": Part XI of the Law of the Sea Convention and Third States: Some General Observations, in Cambridge Law Journal, 1989, 85-87, 94. Elle a de toute façon été rejetée par la CIJ. V. notamment : CIJ, Délimitation de la frontière maritime dans la région du Golfe du Maine (Canada/Etats Unis d'Amérique), Arrêt, 12 Oct. 1984, § 94.

${ }^{61}$ Sur la question : Robert Kolb, L'interprétation de l'article 121, paragraphe 3, de la Convention de Montego Bay sur le droit de la mer, in AFDI, 1994, 876-909 ; Syméon Karagiannis, Les rochers qui ne se prêtent pas à l'habitation humaine ou à une vie économique propre et le droit de la mer, in RBDI, 1996, 559-624.

${ }^{62}$ CIJ, Délimitation maritime et questions territoriales entre Qatar et Bahrë̈n (Qatar c. Bahreïn), arrêt, 16 Mars 2001, § 195 (article 121 paragraphe 1 ) et $\S 185$ (article 121 paragraphe 2).

${ }^{63}$ V. notamment : Barbara Kwiatkowska, Alfred H. A. Soons, Entitlement to Maritime Areas of Rocks Which Cannot Sustain Human Habitation or Economic Life of Their Own, in Netherlands Yearbook International Law, 1990, 139, 174-180 : Yoshifumi Tanaka, The International Law of the Sea, Cambridge, Cambridge University Press, 2012, 64-67.

${ }^{64}$ V. sur la question notamment : Sean Murphy, International Law Relating to Islands, in RCADI, Vol. 386, 9265, 50 .
} 
disposition $^{65}$. Dans l'affaire du Différend territorial et maritime (Nicaragua c. Colombie), la Cour considère que le régime juridique de l'article 121 est unique. Formant un tout indivisible, elle en conclut qu'il fait partie du droit international coutumier. Si la conclusion n'est pas forcément contestable, la motivation prête en revanche le flanc à la critique. Elle est beaucoup trop lapidaire et ne permet pas, une bonne fois pour toute, de clore le débat. Il est vrai que le rôle de la Cour n'est pas de jouer au législateur mais de régler des différends. Aussi avait-elle bien pris soin de préciser que, dans la présente affaire, les parties s'étaient entendues pour reconnaître à la disposition en cause un caractère coutumier ${ }^{66}$. Cette constatation permet indiscutablement de rendre plus acceptable sa conclusion à l'égard des destinataires de l'arrêt. Il n'en demeure pas moins que la seule volonté des parties au différend ne saurait avoir pour effet de transformer une disposition conventionnelle en norme coutumière. Compte tenu de son insuffisante motivation, la Cour n'a pas mis un terme à la controverse. Après le prononcé de l'arrêt de la CIJ, certains continuent de s'interroger sur le caractère coutumier du troisième paragraphe de l'article $121^{67}$ tandis que d'autres au contraire le défendent ${ }^{68}$.

\footnotetext{
${ }^{65}$ V. notamment : Jonathan I. Charney, Rock that Cannot Sustain Human Habitation, in American Journal of International Law, 1999, 863-878, 872 pour qui : "Yet the negotiations at UNCLOS III that produced the Convention, the widespread participation by states in those negotiations, the large number of states parties to the Convention from all sectors of the international community, the denial of a right to make reservations to this article and the widely held view that the normative provisions of the Convention reflect general international law-all support the conclusion that Article 121(3) is general international law applicable to the entire continental shelf regime. Moreover, the text of the article (whose genesis can be found in the 1970s) was settled early in the negotiations. Statements made by states accepting the normative provisions of the Convention as general international law lend additional support to this view. Thus, Article 121 (3) is law for all states with respect to the seaward limits of the continental shelf. Because it is also an inherent part of the regime of the exclusive economic zone, the same case can be made that Article 121 (3) is binding on states that are not parties to the LOS Convention with respect to that regime".

${ }^{66}$ Dans l'affaire de la Délimitation maritime entre le Nicaragua et la Colombie, les parties reconnaissaient une valeur coutumière aux articles 74, 76 § 1, 83 et 121 de la Convention : Arrêt, § 116-117.

${ }^{67}$ V. notamment Yoshifumi Tanaka selon qui "there are serious doubts whether UNCLOS Article 121(3) can be thought to represent part of customary international law. Even if this provision became part of customary international law, its precise meaning would remain obscure": Reflections on the territorial and maritime dispute between Nicaragua and Colombia before the International Court of Justice, in LJIL, 2013, 909-931, 913. V.
} aussi 930 .

${ }^{68}$ V. notamment Sean Murphy selon qui, "[t]he lack of an exception in the 1958 Convention should be understood partly in light of that Convention's relatively modest approach to the outer limit of the continental shelf, given the technology of the time, as compared with the 1982 LOS Convention. By the time the 1982 Convention was being adopted, many States and commentators viewed Article 121 as reflecting customary international law. For example, the conciliation commission with respect to the Iceland/Norway Jan Mayen dispute concluded in 1981, just prior to adoption of the Convention, that "this article reflects the present status of international law on this subject" [Conciliation Commission on the Continental Shelf Area between Iceland and Jan Mayen: Report and recommendations to the governments of Iceland and Norway, Decision of June 1981, Reports of International Arbitral Awards, Vol. XXVII, p. 10 (...) ]. Further, as of 2016, nearly 170 States are parties to the LOS Convention, leaving relatively few States outside the convention regime, and thus suggesting widespread acceptance by States to the rules set forth in Article 121. Non-parties to the LOS Convention have not asserted that Article 121, paragraph 3, is inconsistent with customary international law (...). Any contemporary practice by States Parties to the LOS Convention that advances or accepts claims to extended maritime zones based on very small, uninhabited islands does not reflect upon the status of customary international law; it reflects either an interpretation or a violation of paragraph 3. State practice by non-Parties advancing or accepting such claims also likely reflects an interpretation or violation of a customary international law obligation that tracks paragraph 3 (...) To the extent that there are any States or commentators that continue to believe that paragraph 3 does not reflect customary international law, it seems likely that, under the influence of the Court's 2012 judgment and the LOS Convention itself, such views will eventually dissipate, leading to a consensus that all of Article 121 is settled customary international law" : International Law Relating to Islands, 
Mutatis mutandis, c'est une difficulté que l'on rencontre également à propos de l'article 76 de la CNUDM. Comme il a été mentionné précédemment, la Cour internationale de Justice a affirmé, à juste titre, que le premier paragraphe reflétait le droit coutumier. Elle a en revanche refusé de se prononcer sur les autres paragraphes de cette disposition. Dans l'affaire du Différend territorial et maritime (Nicaragua c. Colombie), la Cour estime que l'article 76 est opposable au Nicaragua dans son intégralité. Il en résulte que cet Etat doit impérativement respecter la procédure qui y est consignée s'il souhaite fixer les limites extérieures à son plateau continental s'étendant au-delà de 200 milles marins. C'est une obligation qui découle de sa qualité de partie à la Convention. Cependant, là où le bât blesse, dans le raisonnement de la Cour, est qu'elle impose au Nicaragua de respecter une disposition de la CNUDM dans le cadre d'une délimitation maritime impliquant un Etat tiers à la convention. Dès lors que la Colombie n’y est par partie, seules les dispositions reflétant le droit coutumier auraient dû trouver à s’appliquer. Or, comme il a été précisé, la CIJ n’a pas jugé utile de s'interroger sur la nature coutumière des dispositions en cause ${ }^{69}$. Se référant à la volonté consacrée dans le préambule d'instaurer un « ordre juridique des mers et des océans ", la Cour estime que ce n'est pas parce la Colombie n'est pas partie à la Convention, que le Nicaragua doit être exonéré des obligations contenues à l'article $76^{70}$. Comme l'observe Lucie Delabie, « une telle motivation, qui n'est pas exempte de critiques, masque mal les considérations d'opportunité qui ont guidé les juges. Affirmer que le droit applicable au Nicaragua est l'article 76 dans son ensemble permet d'écarter l'argument du demandeur au prétexte qu'il n'aurait pas respecté la procédure prévue par cette disposition. En effet, la Cour note qu'en l'espèce, le Nicaragua n'ayant transmis que des informations préliminaires à la Commission, elle n'a pas à se prononcer ${ }^{71}$. Plusieurs membres de la Cour ont au demeurant critiqué cet aspect de la décision en ce qu'il remet ni plus ni moins en cause le principe res inter alios $\operatorname{acta}^{72}$. Cette jurisprudence semble compromettre en outre la possibilité pour un Etat non partie à la Convention de faire valoir son droit à un plateau continental au-delà des 200 milles nautiques ${ }^{73}$.

Le 17 mars 2016, la Cour a rendu son arrêt sur les exceptions préliminaires dans l'affaire de la Question de la délimitation du plateau continental entre le Nicaragua et la Colombie au-delà de 200 milles marins de la côte nicaraguayenne. A titre d'exception préliminaire, la Colombie contestait la recevabilité de la requête nicaraguayenne dans la mesure où le Nicaragua n'avait pas obtenu de recommandation de la part de la Commission

in RCADI, Vol. 386, 9-265, 50-51. V. également : Malcolm Evans, Maritime Boundary Delimitation, in D. Rothwell et al. (eds.), The Oxford Handbook of the Law of the Sea, Oxford, OUP, 2016, 254-279, 263.

${ }^{69}$ Le juge Bennouna avait pourtant expressément posé la question aux parties lors des audiences : "Les règles posées à l'article 76 de la Convention des Nations Unies de 1982 sur le droit de la mer, pour la détermination de la limite extérieure du plateau continental au-delà des 200 milles marins, peuvent-elles être considérées aujourd'hui comme ayant le caractère de règles de droit international coutumier ? " : CR 2012/17, Vendredi 4 Mai 2012, 41. Tandis que le Nicaragua considérait que les paragraphes 1 à 7 reflétait le droit coutumier, la Colombie le contestait pour les paragraphes 4 à 9 : Arrêt, \$116-117. Pour une analyse du caractère coutumier de l'article 76 : Tullio Treves, Codification du droit international et pratique des Etats dans le droit de la mer, in RCADI, 1990, Vol. 223, 9-302, 89-103 et UNCLOS and Non-Party States before the International Court of Justice, in C. Espósito, J. Kraska, H.N. Scheiber, M.-S. Kwon (ed.), Ocean Law and Policy. 20 Years under UNCLOS, Leiden/Boston, Brill/Nijhoff, 2016, 367-378, 372-375.

${ }^{70}$ CIJ, Différend territorial et maritime (Nicaragua c. Colombie), Arrêt, 19 Nov. 2012, § 126.

${ }^{71}$ Lucie Delabie, Le fragile équilibre entre prévisibilité juridique et opportunité judiciaire en matière de délimitation maritime : l'arrêt de la Cour internationale de Justice du 19 novembre 2012 dans l'affaire du Différend territorial et maritime (Nicaragua c. Colombie), in AFDI, 2012, 223-252, 237.

${ }^{72}$ V. en ce sens : Déclaration Cot, § 19 ; Déclaration Mensah, § 2 et 3. V. également Déclaration Donoghue, $\S$ 26.

${ }^{73}$ V. Déclaration Mensah, § 6. 
des limites du plateau continental ; ce que la Colombie jugeait contraire à l'article 76, paragraphe 8 de la CNUDM. Afin d'écarter cette exception, la Cour précise que la communication à la Commission des informations sur les limites de son plateau continental au-delà de 200 milles marins est une obligation qui pèse sur le Nicaragua, en tant qu'Etat partie à la CNUDM (obligation dont il s'est en l'espèce acquittée) en revanche, l'adoption d'une recommandation par la Commission, après examen de ces informations, est une prérogative de celle-ci. La Cour en conclut que « dès lors que la délimitation du plateau continental au-delà de 200 milles marins peut s'effectuer indépendamment de la recommandation de la Commission, celle-ci n'est pas un prérequis pour qu'un Etat partie à la CNUDM puisse demander à la Cour de régler un différend avec un autre Etat relatif à une telle délimitation ${ }^{74}$. En conséquence, la Cour rejette l'exception préliminaire d'irrecevabilité. Ce qui n'est pas forcément en totale harmonie avec les décisions passées. En effet, dans l'affaire du Différend territorial et maritime entre le Nicaragua et le Honduras dans la mer des Caraïbes, elle avait affirmé que toute prétention devait être «examinée par » la Commission $^{75}$ ce qui pouvait laisser entendre qu'une recommandation était obligatoire ${ }^{76}$. Cette affirmation avait de plus été reprise dans l'affaire du Différend territorial et maritime entre le Nicaragua et la Colombie ${ }^{77}$.

Par ailleurs, toujours dans cet arrêt du 17 mars 2016, il est intéressant de remarquer qu'à l'appui de son argumentation, la Colombie s'est expressément fondée sur l'article 76 de la Convention dans son intégralité alors que c'est un Etat tiers et que le caractère coutumier des dispositions en question n'a pas été établi (à l'exception du premier paragraphe) ${ }^{78}$. L'exception d'irrecevabilité aurait pu être rejetée sur ce seul fondement ${ }^{79}$. Quoi qu'il en soit, il sera intéressant de voir le sort que la Cour va réserver à cette affaire au fond et notamment la façon dont elle va s'en sortir pour concilier, d'une part, la Convention de Montego Bay et la coutume ainsi que, d'autre part, les droits et obligations des Etats parties à la Convention avec ceux des Etats tiers.

\footnotetext{
${ }^{74}$ CIJ, Question de la délimitation du plateau continental entre le Nicaragua et la Colombie au-delà de 200 milles marins de la côte nicaraguayenne (Nicaragua c. Colombie), Arrêt, 16 Mars 2016, § 114. Cette position a été confirmée par la suite: CIJ, Délimitation maritime dans l'Océan indien (Somalie c. Kenya), exceptions préliminaires, 2 Fév. 2017, § 67.

75 «[T] oute prétention relative à des droits sur le plateau continental au-delà de 200 milles doit être conforme à l'article 76 de la CNUDM et examinée par la Commission des limites du plateau continental constituée en vertu de ce traité »: CIJ, Différend territorial et maritime entre le Nicaragua et le Honduras dans la mer des Caraïbes (Nicaragua c. Honduras), Arrêt, 8 Oct. 2007, § 319.

${ }^{76}$ V. en ce sens Déclaration Donoghue, § 50. De manière générale sur la question : Massimo Lando, Delimiting the Continental Shelf Beyond 200 Nautical Miles at the International Court of Justice: The Nicaragua v. Colombia Cases, in Chinese Journal of International Law, 2017, 137-173.

${ }^{77}$ CIJ, Différend territorial et maritime (Nicaragua c. Colombie), Arrêt, 19 Nov. 2012, § 126.

${ }^{78}$ L'attitude de la Colombie n'est cependant pas sans contradiction. Si devant la CIJ, elle se réfère à l'article 76 de la CNUDM, devant les Nations Unies elle insiste au contraire sur son Statut de tiers à la Convention lorsqu'il s’agit de protester contre les revendications du Nicaragua au bénéfice d'un plateau continental étendu. V. notamment Annexe à la note verbale datée du 6 février 2014 adressée au Secrétaire général par la Mission permanente de la Colombie auprès de l'Organisation des Nations Unies, 5 Fév. 2014, A/68/743 dans laquelle elle réaffirme qu'elle «n'est pas partie à la Convention des Nations Unies sur le droit de la mer. En conséquence, la demande du Nicaragua ne peut être opposée à la Colombie et n’a aucune incidence sur les droits que celle-ci exerce sur son plateau continental. La Colombie réitère qu'elle n’a pas consenti à cette procédure ».

${ }^{79}$ Dans son opinion individuelle, le juge Owada précise que « les dispositions pertinentes de l'article 76 de la CNUDM ne peuvent être invoquées à l'égard du Nicaragua par la Colombie, à moins que celle-ci ne puisse établir qu’il s’agit de règles du droit international coutumier » : § 39. V. également Déclaration Robinson, § 3-5 et 16 .
} 
Nonobstant l'adoption de la CNUDM, la coutume conserve une certaine importance en droit de la mer. Comme il a été vu, il arrive qu'aujourd'hui encore la Cour internationale de Justice tranche des différends se rapportant au droit de la mer en se fondant uniquement sur le droit coutumier. Mais, même lorsqu'elle se fonde sur la coutume, la CNUDM n'est jamais très loin tant celle-ci reflète le droit coutumier.

Ainsi que l'a expressément souligné la CIJ, la Convention des Nations Unies sur le droit de la mer est un texte de compromis ${ }^{80}$. Il en résulte qu'un certain nombre de ses dispositions sont évasives et laissent à l'interprète une certaine marge d'appréciation ${ }^{81}$. C'est ce que confirme d'ailleurs la Cour lorsqu'elle relève que la Convention "se borne à énoncer une norme et laisse aux Etats ou au juge le soin de lui donner un contenu précis ${ }^{82}$. C'est de la sorte par exemple qu'elle a établi progressivement une méthode de délimitation maritime en trois étapes consistant à établir un tracé provisoire d'une ligne d'équidistance puis à contrôler cette ligne à la lumière d'une série de circonstances pertinentes et enfin à vérifier que le tracé retenu n’est pas inéquitable. Cette méthode a été systématisée de manière très pédagogique dans l'arrêt Délimitation maritime en mer Noire ${ }^{83}$. De la même façon, depuis l'affaire du Golfe du Maine ${ }^{84}$, les Etats demandent généralement à la Cour de délimiter, par une ligne unique, le plateau continental et la ZEE alors même que la Convention ne l'impose pas $^{85}$. Cette frontière maritime unique trouve son origine dans la pratique des Etats et dans l'accueil favorable que lui a réservé la Cour. Par ses multiples interprétations des dispositions conventionnelles ou de leurs reflets coutumiers, la Cour internationale de Justice contribue de manière significative à enrichir le droit de la mer. Mutatis mutandis, il en va de même du Tribunal international du droit de la mer ou bien encore des tribunaux arbitraux ${ }^{86}$. Ces

\footnotetext{
${ }^{80}$ CIJ, Délimitation de la frontière maritime dans la région du golfe du Maine (Canada/Etats-Unis d'Amérique), Arrêt, 12 Oct. 1984, § 94.

${ }^{81}$ Celle-ci est en outre renforcée par le fait que la convention renvoie très fréquemment à l'équité. V. par exemple les articles 83, 140, 160 et $\S 2$ f) i.

${ }^{82}$ CIJ, Plateau continental (Jamahiriya arabe libyenne/Malte), Arrêt, 3 Juin 1985, § 28. D’aucuns n’ont d'ailleurs pas hésité, pour certaines dispositions de la convention, à employer le terme de "décodification »; décodification par rapport aux conventions de 1958. C’est notamment le cas du professeur Treves qui a justement affirmé qu' « on a voulu balayer le terrain pour le laisser libre au juge et aux parties » : Tullio Treves, intervention faite au cours des débats du colloque SFDI, La codification du droit international, Paris, Pedone, 1999, 327.

${ }^{83}$ CIJ, Délimitation maritime en mer Noire (Roumanie c. Ukraine), Arrêt, 3 Fév. 2009, passim. § 115-122. V. notamment: Alain Pellet, Benjamin Samson, La délimitation des espaces marins, in M. Forteau, J.-M. Thouvenin (dirs.), Traité de droit international de la mer, Paris, Pedone, 2017, 565-623, 593-608.

${ }^{84}$ CIJ, Délimitation de la frontière maritime dans la région du golfe du Maine (Canada/Etats Unis d'Amérique), 12 Oct. 1984, § 26.

${ }^{85}$ Ainsi que l'observe le Président Tomka, « à compter de cette affaire, les parties à des différends en matière de délimitation maritime portés devant la Cour ont, pour la plupart, sollicité l'établissement d'une ligne unique pour diviser leurs droits maritimes respectifs (...). A cet égard, on notera que la notion de frontière maritime unique n’apparaît nulle part dans la convention des Nations Unies sur le droit de la mer (...). De fait, elle trouve son origine dans la pratique des Etats, évolution dont la Cour s'est félicitée et qu'elle a intégrée dans sa jurisprudence, tout en continuant à confirmer d'autres normes conventionnelles et coutumières applicables » : Discours de S. Exc. Peter Tomka, président de la Cour internationale de Justice, devant la sixième commission de l’Assemblée générale, 2 novembre 2012, 4.

${ }^{86}$ En droit de la mer, « la jurisprudence n’a pas seulement une fonction d'interprétation ou même d'identification et de précision des règles de droit mais joue un rôle beaucoup plus large que celui qu'on considère d'habitude comme celui d'une source auxiliaire du droit international. En fait, la jurisprudence est dans ce domaine en rapport direct avec la coutume internationale, permettant sa constatation de manière plus précise, comme elle est aussi étroitement liée aux règles conventionnelles qui surgissent des processus de codification et de développement progressif du droit international ayant fréquemment anticipé ou orienté ces développements » :
} 
différents organes juridictionnels exercent indiscutablement un rôle de suppléance normative qu'ils maquillent plus ou moins bien derrière l'opération d'interprétation des traités ou de révélation de la coutume.

Le juge joue évidemment un rôle fondamental dans l'établissement de la coutume. La CIJ ne s'en cache du reste pas. Dans son arrêt Jan Mayen, elle n'a pas hésité pas à évoquer le «droit coutumier du plateau continental tel qu’il s’est développé dans la jurisprudence » ${ }^{87}$. Cette phrase est extrêmement lourde de sens. La Cour reconnaît ainsi expressément l'existence de règles coutumières qui « découlent directement de la seule intervention du juge et non pas de coutumes qu'il se serait contenté de constater et qui ne devraient guère à son action ${ }^{88}$. Les parties ne sont évidemment pas sans l'ignorer. C'est ce qui explique que dans l'affaire du Différend frontalier et maritime, par exemple, le Nicaragua et la Colombie s'étaient entendu pour considérer que le droit applicable en la présente affaire était le droit international coutumier tel qu'il ressort de la jurisprudence de la Cour, du Tribunal international du droit de la mer et des tribunaux arbitraux internationaux ${ }^{89}$.

Francisco Orrego Vicuña, Le rôle de la jurisprudence internationale, in M. Forteau, J.-M. Thouvenin (dirs.), Traité de droit international de la mer, Paris, Pedone, 2017, 77-94, 77.

${ }^{87}$ CIJ, Délimitation maritime dans la région située entre le Groenland et Jan Mayen (Danemark c. Norvège), Arrêt, 14 Juin 1993, § 51.

${ }^{88}$ Laurent Lucchini, Michel Voelckel, Droit de la mer, Paris, Pedone 1996, 2 t., vol. I, 202. V. également sur la question: Guillaume Le Floch, La coutume dans la jurisprudence de la Cour internationale de Justice en droit de la mer, in Revue Juridique de l'Ouest, 2001, 535-573, 565.

${ }^{89}$ CIJ, Différend territorial et maritime (Nicaragua c. Colombie), Arrêt, 19 Nov. 2012, § 114. 